\title{
AVALIAÇÃO DE TAMANHO DE BOLHAS EM COLUNA DE FLOTAÇÃO POR ANÁLISE DE IMAGEM USANDO OS SOFTWARES IMAGEJ E APBS
}

\author{
Renata Nigri de Almeida ' \\ Estevão Gomes da Cruz Barreto ${ }^{2}$ \\ Hudson Jean Bianquini Couto ${ }^{1,3}$
}

\section{Resumo}

O tamanho médio e a distribuição de tamanho de bolhas (DTB) são variáveis importantes no processo de flotação, pois influenciam diretamente na eficiência de coleta e no transporte das partículas. Neste contexto, o objetivo deste trabalho foi medir e avaliar o tamanho médio e a DTB geradas em uma coluna de flotação em função de algumas variáveis importantes do processo de flotação. Para captura e visualização das bolhas foi utilizado o equipamento Anglo Platinum Bubble Sizer (APBS). As imagens das bolhas capturadas foram processadas com utilização de dois softwares, o APBS e o ImageJ, de forma a comparar os resultados obtidos por ambos. Além de calcular o diâmetro médio e a DTB das bolhas, foi possível realizar medidas de holdup de gás $\left(\varepsilon_{\mathrm{g}}\right)$ e da concentração crítica de coalescência $(C C C)$ para o Flotanol DI4, agente tensoativo avaliado no trabalho. Os resultados obtidos de $\varepsilon_{\mathrm{g}}$ e CCC apontaram para uma boa concordância com os valores encontrados na literatura. Considerou-se o ImageJ mais confível para o processamento de imagem e análise do tamanho de bolha devido ao seu maior controle das etapas de processamento.

Palavras-chave: Coluna de flotação; Tamanho de bolha; Análise de imagens.

\section{EVALUATION OF BUBBLE SIZE IN A FLOTATION COLUMN BY IMAGE ANALYSIS USING THE SOFTWARES IMAGEJ AND APBS}

\begin{abstract}
The average size and the bubble size distribution (BSD) are important variables in the flotation process, as they influence the efficiency on collecting and transporting the particles. In this context, the aim of this work was to measure and evaluate the average size and BSD generated in a flotation column as a function of some important variables of the flotation process. The Anglo Platinum Bubble Sizer (APBS) was used to capture and visualize the bubbles. The images of the captured bubbles were processed using two softwares, the APBS and the ImageJ (public domain), in order to compare the results obtained by both. In addition to calculating the diameter of the bubbles, it was possible to perform measurements of gas holdup $\left(\varepsilon_{g}\right)$ and critical coalescence concentration (CCC) for Flotanol DI4, surfactant evaluated. The results obtained from $\varepsilon_{g}$ and $C C C$ showed a good agreement with the values found in the literature. The Imagej software was considered more reliable for image processing and bubble size analysis due to its control of the processing step.
\end{abstract}

Keywords: Column flotation; Bubble size; Image analysis.

\section{INTRODUÇÃO}

O processo de flotação vem sendo utilizado desde o início do século $X X$ na separação seletiva de minerais, e mais atualmente, no tratamento de efluentes em escala industrial. Esse processo físico-químico consiste na adesão de bolhas de ar introduzidas no sistema à fase dispersa (partículas), sendo o conjunto formado bolha-partícula carreado para a superfície da célula de flotação onde ocorre a recuperação das partículas. É um dos processos mais utilizados industrialmente na concentração mineral por apresentar um aproveitamento de minérios complexos e de baixos teores de forma econômica e com bons rendimentos metalúrgicos [I].

'Centro de Tecnologia Mineral - CETEM, Rio de Janeiro, RJ, Brasil.

${ }^{2}$ Universidade Federal do Rio de Janeiro - UFRJ, Rio de Janeiro, RJ, Brasil.

3Instituto Federal do Rio de Janeiro - IFRJ, Rio de Janeiro, RJ, Brasil. E-mail: hudson.couto@ifrj.edu.br 
Em relação aos diferentes processos de flotação existentes, os principais são: flotação por ar induzido (FAl) e flotação por ar dissolvido (FAD). Estes processos se diferenciam principalmente pela forma como $o$ ar é introduzido ao sistema. O processo de FAl ocorre quando o ar é induzido ao sistema através de dispositivos como distribuidores porosos, ejetores gás-líquido (sistema tipo tubo de Venture), sistemas de injeção de ar (tais como impelidores rotativos ou bicos especiais), dentre outros [2].

$\mathrm{Na}$ área de processamento mineral, existe um grande interesse na recuperação de finos, principalmente daqueles perdidos na corrente de descarte de circuitos de flotação convencional, considerados lamas, constituindo um dos principais problemas desse processo. Um dos principais motivos para isso é que os equipamentos de flotação convencional geram bolhas com diâmetro na faixa de $600-2500 \mu \mathrm{m}$, consideradas grandes para a separação efetiva de partículas finas. A ausência de bolhas pequenas e médias $(10-600 \mu \mathrm{m})$ em células convencionais de flotação é o principal motivo da ineficiência na recuperação de finos por esse processo, levando a consideráveis perdas, especialmente para partículas menores que $13 \mu \mathrm{m}[2,3]$.

Um dos grandes avanços do processo de flotação foi o surgimento da flotação em coluna, visando resolver parte dos problemas de perdas de partículas finas. Outras vantagens da flotação em coluna com relação à flotação em célula mecânica são: uma maior relação altura/diâmetro (H/D), aumentando a probabilidade de contato bolha-partícula e a maior seletividade pela aplicação de água de lavagem, diminuindo $o$ arraste hidrodinâmico de partículas hidrofilicas para a espuma [4].

Nesse contexto, o tamanho e a distribuição de tamanho de bolhas (DTB) são parâmetros importantes no processo de flotação devido a seu efeito na eficiência de coleta e de transporte das partículas, exigindo uma variação extensa da DTB para diversos tamanhos de partícula. Nas colunas de flotação, o tamanho médio de bolhas está situado na faixa de 500 a $2000 \mu \mathrm{m}$ [4].

Quanto menor o tamanho das bolhas geradas, maior é a área interfacial de contato bolha-partícula e maior é a eficiência do processo, considerando um mesmo holdup de gás. Entretanto, bolhas de tamanhos muito reduzidos apresentam uma velocidade de ascensão baixa, podendo ser inferior à velocidade descendente da polpa, acarretando, em consequência, perdas de partículas hidrofóbicas coletadas no fluxo de material não flotado. Nesse contexto, existe um tamanho médio ideal de bolhas, que poderá ser ajustado por meio de variáveis operacionais ou por meio da adição de soluções tensoativas [4].

Diversos autores reportam a importância de se determinar e controlar o tamanho de bolhas, dentre os diversos métodos existentes, tais como a utilização de correlações fluidodinâmicas, medidas de eletro resistividade, difração laser, análise de imagem, dentre outras [4-12]. Entre esses métodos destacam-se aqueles com base na captura e análise de imagens digitais utilizando visores externos, mostrando-se uma solução promissora para medição do tamanho de bolhas em células de flotação industriais.

É importante destacar que as técnicas baseadas na obtenção de imagens digitais podem ser classificadas em estáticas e dinâmicas. Na primeira as bolhas amostradas da célula de flotação são transportadas até uma câmara de visualização horizontal, onde são imobilizadas e fotografadas por I-2 segundos com o auxílio de um microscópio ótico. Como exemplo desta técnica cita-se a denominada LTM-BSizer [10, 13], cuja vantagens destacadas pelos autores são a melhor qualidade das imagens e a capacidade de medir distribuições de tamanho de bolha com maior amplitude. Como desvantagem é preciso fazer diversas amostragens para se obter um número estatisticamente significativo de bolhas e frequentemente ocorre a formação de aglomerados de bolhas (clusters), que são atenuados com a utilização de algoritmos de segmentação. Na técnica dinâmica a obtenção de imagens digitais é realizada em visores externos verticais, geralmente em plano inclinado para o melhor espalhamento das bolhas em movimento. Nesta técnica várias fotografias podem ser obtidas em pouco tempo para obtenção de um número suficientemente grande de bolhas para análise. Como principal desvantagem cita-se a maior dificuldade de medição de bolhas de menor tamanho (principalmente $<100 \mu \mathrm{m}$ ), em função da menor qualidade de imagem dessas bolhas, o que vem sendo amenizado com as câmeras CCD atuais.

$O$ presente trabalho tem como objetivo avaliar o tamanho médio e a distribuição do tamanho de bolhas (DTB) geradas em uma coluna de flotação de 3" de diâmetro do Centro de Tecnologia Mineral (CETEM), utilizando a técnica dinâmica (equipamento Bubble Sizer) de obtenção de imagens digitais. Algumas variáveis importantes do processo de flotação, a exemplo da concentração de espumante e velocidade superficial do ar, foram avaliadas utilizando-se dois softwares de processamento de imagens digitais (Image) e APBS) para comparação dos resultados obtidos.

\section{MATERIAIS E MÉTODOS}

Os ensaios deste trabalho foram realizados no CETEM, em uma coluna de 7,6 cm (3") de diâmetro em um sistema bifásico líquido/gás (solução aquosa/bolhas de ar), sem a presença de minério. Compreenderam a avaliação da dosagem do espumante $\left(C_{\mathrm{f}}\right)$ e da velocidade superficial do $\operatorname{ar}\left(\mathrm{J}_{\mathrm{g}}\right)$, sobre os parâmetros tamanho médio de bolha (diâmetro médio de Sauter, $\left.D_{3,2}\right)$ e holdup de gás $\left(\varepsilon_{g}\right)$.

\section{I Reagentes}

Para os estudos de determinação do tamanho de bolhas e de holdup de gás foi utilizado o espumante Flotanol DI4, um polipropileno glicol metileter, fornecido pela Clariant. Foram utilizadas dosagens deste reagente nas concentrações de $2,5,5,10,20$ e $50 \mathrm{mg} / \mathrm{L}$. 


\subsection{Coluna de Flotação}

Os ensaios foram realizados em uma coluna de flotação de bancada, com $7,6 \mathrm{~cm}$ de diâmetro interno e $159 \mathrm{~cm}$ de altura, construída em PVC transparente e volume total de 7,2 L, fabricada pela Eriez Minerals Flotation Group (Canadá). O aparato é constituído também por um sistema de controle de nível, rotâmetros para controle da vazão de água de lavagem e de ar, e bombas peristálticas para alimentação e retirada de produtos. O fluxo de bolhas de ar foi produzido por um aerador tipo tubo poroso, instalado na parte inferior da coluna. As condições operacionais dos ensaios estão descritas na Tabela I.

A velocidade superficial de alimentação de líquido $\left(J_{f}\right)$ e do $\operatorname{ar}\left(J_{g}\right)$ foram obtidas pela razão entre as respectivas vazões de alimentação de líquido $\left(Q_{f}\right)$ e do $\operatorname{ar}\left(Q_{g}\right)$ pela área de seção transversal da coluna $\left(A_{c}\right)$.

\subsection{Procedimento para Visualização e Captura das Bolhas}

A Figura la mostra o desenho esquemático do sistema montado para os ensaios de determinação do tamanho de bolha e a Figura Ib, a foto da coluna de flotação acoplada ao APBS utilizada nos ensaios.

Tabela I. Condições operacionais dos ensaios para determinação do tamanho de bolha na coluna de flotação

\begin{tabular}{lc}
\hline \multicolumn{1}{c}{ Parâmetros } & Condições \\
\hline Vazão de alimentação da coluna $\left(\mathrm{Q}_{\mathrm{f}}\right)$ & $0,28 \mathrm{~L} / \mathrm{min}$ \\
Velocidade superficial de alimentação $\left.\mathrm{J}_{\mathrm{f}}\right)$ & $0,10 \mathrm{~cm} / \mathrm{s}$ \\
Vazão de ar & $0,68-3,42 \mathrm{~L} / \mathrm{min}$ \\
Velocidade superficial do ar $\left(\mathrm{J}_{\mathrm{g}}\right)$ & $0,25-1,25 \mathrm{~cm} / \mathrm{s}$ \\
Dosagens do espumante Flotanol D I4 $\left(\mathrm{C}_{\mathrm{f}}\right)$ & $2,5-50 \mathrm{mg} / \mathrm{L}$ \\
Altura da camada espuma & $20 \mathrm{~cm}$ \\
\hline
\end{tabular}

Para visualização e captura de bolhas foi utilizado o equipamento Anglo Platinum Bubble Sizer (APBS), comercializado pela Stone Three Mining (África do Sul). Esse dispõe de um reservatório de acrílico graduado localizado no topo, um compartimento para instalação da câmera fotográfica e uma fonte de luz. As bolhas geradas na coluna de flotação ascendem por um tubo (tubo amostrador) de acrílico inserido na coluna com $2 \mathrm{~cm}$ de diâmetro e $20 \mathrm{~cm}$ de comprimento posicionado no centro e com um ângulo de $45^{\circ} \mathrm{em}$ relação à coluna e um tubo na vertical com $2,5 \mathrm{~cm}$ de diâmetro $\mathrm{e}$ $20 \mathrm{~cm}$ de comprimento levando as bolhas até a câmara de visualização, a qual possui uma janela de vidro.

A fonte de luz LED é utilizada para garantir o contraste da imagem. A câmara de visualização é posicionada com uma inclinação de 15\%, para permitir o espalhamento das bolhas em uma única camada e minimizar a sobreposição de bolhas, fornecendo um plano inequívoco de foco. As imagens das bolhas foram fotografadas com uma câmera digital Canon modelo PowerShot S95, armazenadas e processadas.

\subsection{Processamento e Análise das Imagens}

O processamento das imagens adquiridas foi realizado por dois diferentes softwares, APBS da Stone Three Mining/Anglo American (versão 2.0) e ImageJ (versão I.48s, de domínio público) [14], com o intuito de comparar os resultados de tamanho de bolhas. O Bubble Sizer é um software do equipamento $A P B S$, onde o algoritmo que utiliza para o processamento das imagens é desconhecido. Fornece informações como, por exemplo, número de bolhas, diâmetros máximo, mínimo, médio de Sauter das bolhas e variância. O Image é um programa livre que pode ser obtido gratuitamente no site https://imagej.nih.gov/ij/, onde sua principal vantagem é a possibilidade de visualizar em

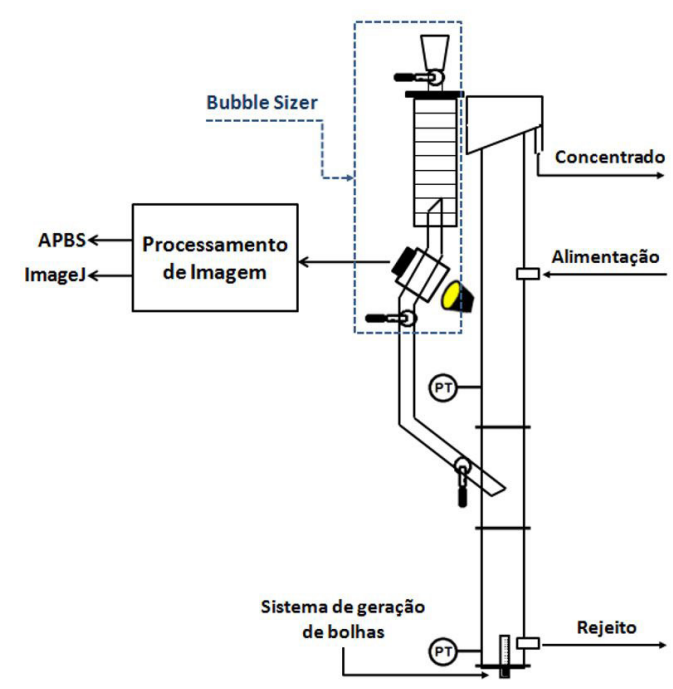

(a)

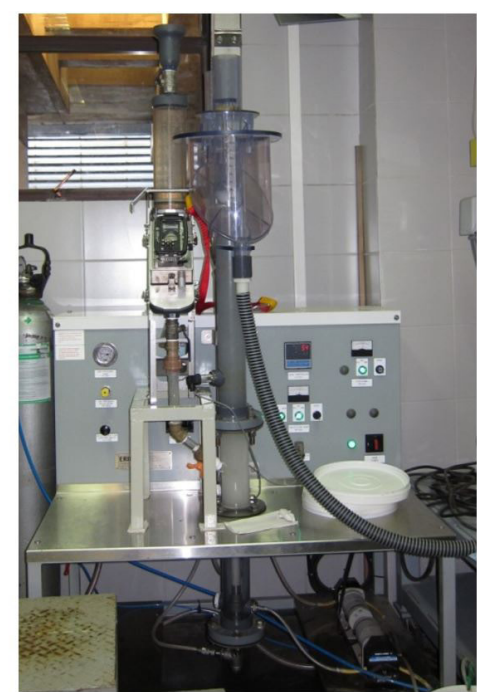

(b)

Figura I. Desenho esquemático do sistema montado (a) e foto da coluna de flotação acoplada ao APBS (b) para os estudos de determinação do tamanho de bolha. 
tempo real o resultado de diferentes técnicas de tratamento de imagens, através de uma interface gráfica. As seguintes etapas foram utilizadas no processamento das imagens: 8-bit (conversão para tons de cinza); threshold (limiarização); fill holes (preenchimento das bolhas demarcadas); watershed (segmentação); identificação e análise das imagens [14]. Os tempos de processamento para ambos os softwares, para o computador utilizado no trabalho, foram muito baixos (menores que I s), por isso não foram avaliados no estudo de comparação.

$\mathrm{Na}$ análise das imagens foi adotado como diâmetro médio o diâmetro médio de Sauter $\left(\mathrm{D}_{3,2}\right)$, definido como o diâmetro médio volume-superfície, conforme apresentado pela Equação I.

$$
\mathrm{D}_{3,2}=\frac{\sum \mathrm{n}_{\mathrm{i}} \cdot \mathrm{d}_{\mathrm{bi}}{ }^{3}}{\sum \mathrm{n}_{\mathrm{i}} \cdot \mathrm{d}_{\mathrm{bi}}{ }^{2}}
$$

Onde $d_{b}$ e $n$ são o diâmetro de bolha e o número de bolhas para classe de tamanho "i”, respectivamente.

\subsection{Determinação do Holdup de Gás}

Foram realizadas medidas de holdup de gás $\left(\varepsilon_{g}\right)$, determinado através de dois transmissores de pressão instalados na lateral coluna, pela diferença de pressão entre esses dois pontos. O holdup foi calculado pela Equação 2, onde $\Delta \mathrm{P}$ se refere à diferença de pressão $(\mathrm{Pa}), \rho_{\mathrm{sl}}$ é a densidade da polpa $\left(\mathrm{kg} / \mathrm{m}^{3}\right)$, $\mathrm{L}$ é a distância entre os transmissores de pressão $(\mathrm{m})$ e g é a aceleração da gravidade $\left(\mathrm{m} / \mathrm{s}^{2}\right)$.

$$
\varepsilon_{\mathrm{g}}=1-\frac{\Delta \mathrm{P}}{\rho_{\mathrm{sl}} \cdot \mathrm{g} \cdot \mathrm{L}}
$$

\subsection{Determinação da Tensão Superficial}

As medidas de tensão superficial das soluções de Flotanol DI 4 foram realizadas com um goniômetro (modelo OCA I5) da DATAPHYSICS, onde a tensão superficial é determinada pelo método da gota pendente.

\subsection{Determinação da Concentração Crítica de Coalescência (CCC)}

Segundo alguns autores $[5,8,15]$, a concentração crítica de coalescência (CCC) de um espumante corresponde a menor concentração que proporciona o menor diâmetro de bolha, sendo um importante parâmetro para comparar o desempenho de diferentes espumantes de flotação. É possível também determinar a CCC através de ajuste dos dados experimentais $\left(\mathrm{D}_{3,2}\right.$ versus $\left.\mathrm{C}_{\mathrm{f}}\right)$ ao modelo de Laskoviski proposto na Equação 3 [16]. Desta forma é possível obter os parâmetros de diâmetro de bolha limite $\left(d_{L}\right)$, redução de diâmetro $(A)$ e constante de decaimento $(B)[15,16]$.

$$
\mathrm{D}_{3,2}=\mathrm{d}_{\mathrm{L}}+\mathrm{A} \cdot \exp \left(-\mathrm{B}_{\mathrm{C}} \mathrm{C}_{\mathrm{f}}\right)
$$

\section{RESULTADOS E DISCUSSÃO}

A Figura 2 mostra as fotografias e a distribuição das bolhas geradas pelo tubo poroso da coluna e a Figura 3a mostra a relação do diâmetro médio de Sauter para diferentes concentrações de Flotanol D I 4 (2,5 até $50 \mathrm{mg} / \mathrm{L}$ ) para uma velocidade superficial constante em $0,5 \mathrm{~cm} / \mathrm{s}$. Para fins de comparação, são apresentados os resultados dos dois softwares utilizados para o processamento das imagens. Os resultados mostram que conforme a concentração do espumante aumenta, o diâmetro das bolhas diminui e, consequentemente, aumenta o número de bolhas, devido à redução do efeito de coalescência, até atingir a estabilização, corroborando com vários trabalhos da literatura $[5,6,8,15,18,19]$.

De acordo com a Figura 3a, observa-se que a partir de concentrações superiores a $7 \mathrm{mg} / \mathrm{L}$, praticamente não houve mudança nos valores do diâmetro médio de Sauter, indicando esta como a CCC deste espumante para as condições operacionais estabelecidas. A partir desta concentração o diâmetro médio ficou estabilizado em, aproximadamente, $0,78 \mathrm{~mm}$ (APBS) e $0,99 \mathrm{~mm}$ (Image/).

A Figura 3b mostra a análise de erro, utilizando duas vezes o desvio padrão e intervalo de confiança de $95 \%$, para os pontos $C_{f}=10,20$ e $50 \mathrm{mg} / \mathrm{L}$. Verifica-se que existe uma diferença entre os valores do $D_{3,2}$ encontrados no APBS e no ImageJ, sendo estes valores sempre maiores no ImageJ, mostrando que o APBS tem uma tendência de subestimar os resultados de tamanho de bolha, mas sem mudanças significativas no valor de CCC do espumante. A tendência de subestimar os valores de tamanho de bolha do APBS pode ser verificada também quando se observa os histogramas da Figura 2, na faixa de tamanho de bolhas entre $\sim 0,2-0,8 \mathrm{~mm}$, em que a quantidade de bolhas nesta faixa para o APBS é sempre maior para o ImageJ, principalmente para as concentrações de 5 e $10 \mathrm{mg} / \mathrm{L}$ de espumante. $\mathrm{Na}$ concentração de $5 \mathrm{mg} / \mathrm{L}$, por exemplo, o APBS identifica bolhas que o ImageJ não identifica nesta faixa de menor tamanho. Sugere-se que estas bolhas mais finas podem ser aprisionadas e acumuladas na janela de visualização e captura das análises digitais que não são descartadas pela rotina de processamento do APBS, que é do tipo "caixa preta", não possibilitando também que o usuário o faça.

$\mathrm{Na}$ Figura $4 \mathrm{a}$ é apresentada uma fotografia de bolhas capturadas pela câmera do equipamento Bubble Sizer e na Figura $4 \mathrm{~b}$ esta mesma fotografia após o processamento com o APBS, onde as bolhas identificadas e medidas são circundadas em verde. É possível observar na ampliação em destaque da Figura $4 \mathrm{~b}$ que algumas bolhas não são identificas e que outras de menor tamanho (pequenos pontos verdes) são identificadas de forma equivocada na etapa de processamento do software.

Os parâmetros do modelo de Laskoviski (Equação 3) foram estimados utilizando o software Statistica, versão I3. I, e estão apresentados na Tabela 2 . Os valores de $d_{L}$ obtidos pela equação ficaram muito próximos aos valores de CCC obtidos de forma gráfica para os dados gerados a partir 

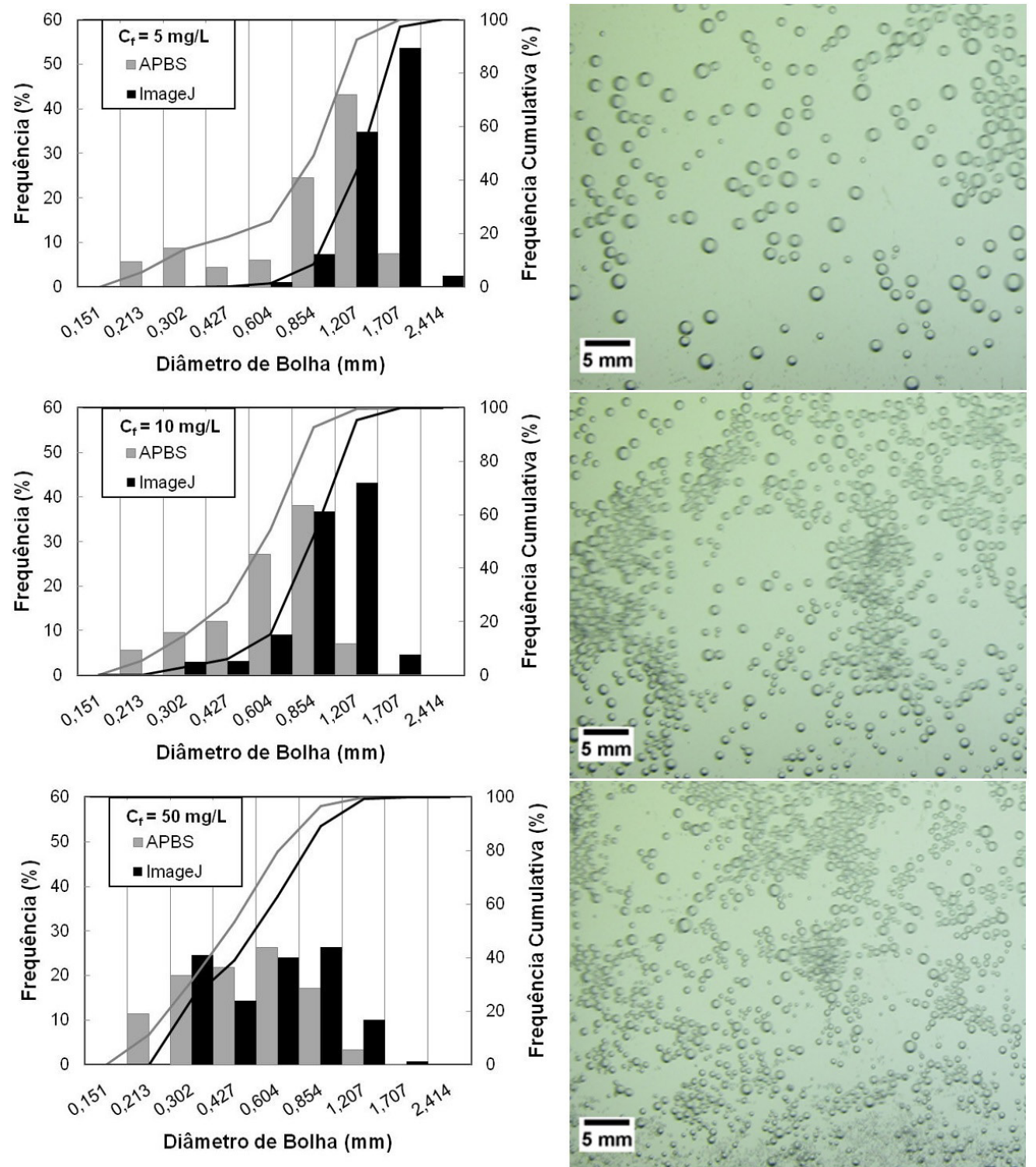

Figura 2. Diâmetro médio de bolha em função da concentração de Flotanol DI 4 e $\int_{\mathrm{g}}$ constante em $0,5 \mathrm{~cm} / \mathrm{s}$.

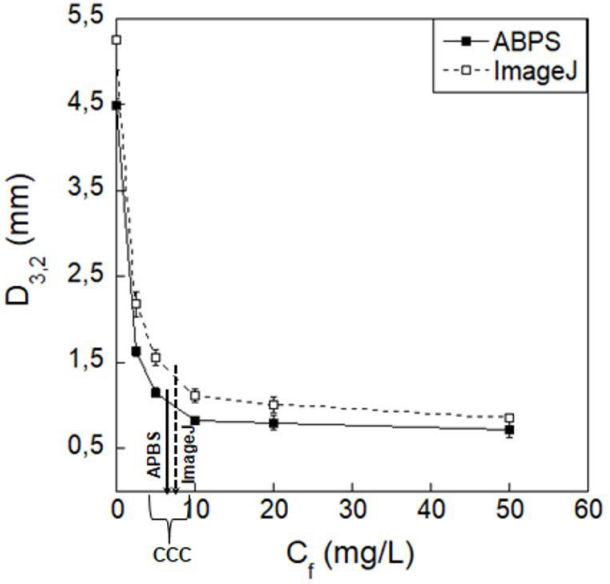

(a)

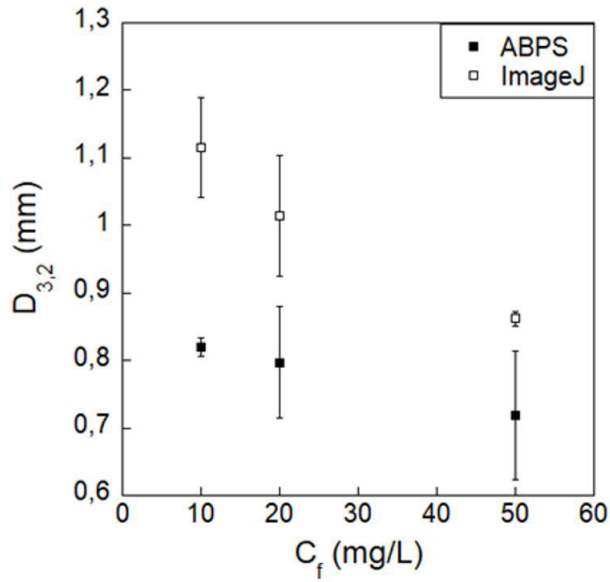

(b)

Figura 3. Diâmetro médio de Sauter em função da concentração do Flotanol DI 4 e J constante em $0,5 \mathrm{~cm} / \mathrm{s}$ (a) e análise de erro para os pontos $C_{\mathrm{f}}=10,20$ e $50 \mathrm{mg} / \mathrm{L}$ (b). Comparação entre os dois softwares utilizados para o processamento de imagem: APBS e ImageJ. 
Tabela 2. Parâmetros de Laskowski dL, A e B

\begin{tabular}{cccc}
\hline & $\begin{array}{c}\text { Diâmetro de bolha limite } \\
(\mathbf{d L})\end{array}$ & $\begin{array}{c}\text { Redução de diâmetro } \\
(\mathbf{A})\end{array}$ & \multicolumn{2}{c}{$\begin{array}{c}\text { Constante de decaimento } \\
\text { (B) }\end{array}$} \\
\hline APBS & 0,800 & 3,686 & 0,565 \\
ImageJ & 1,009 & 4,229 & 0,478 \\
\hline
\end{tabular}

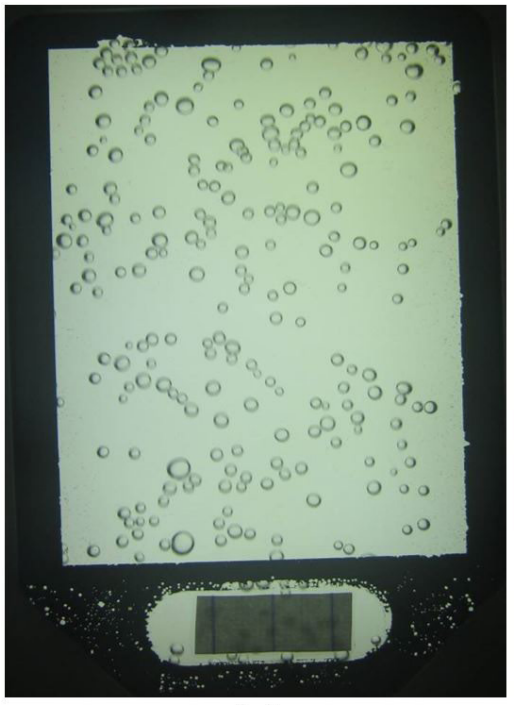

(a)

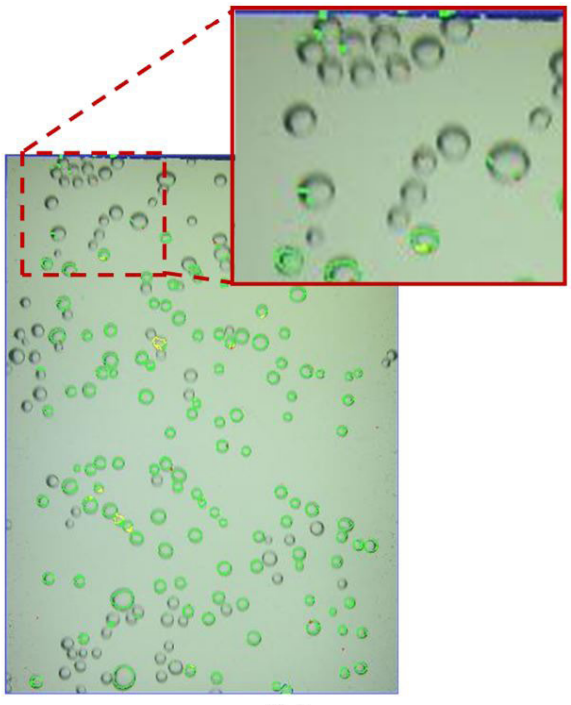

(b)

Figura 4. Fotografia digital obtida na etapa de captura de bolhas na câmara de visualização (a) e a mesma fotografia processada pelo software da APBS, com ampliação de uma área em destaque (b).

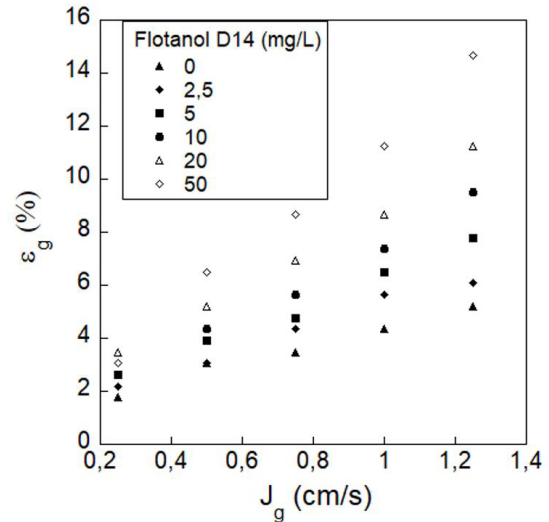

(a)

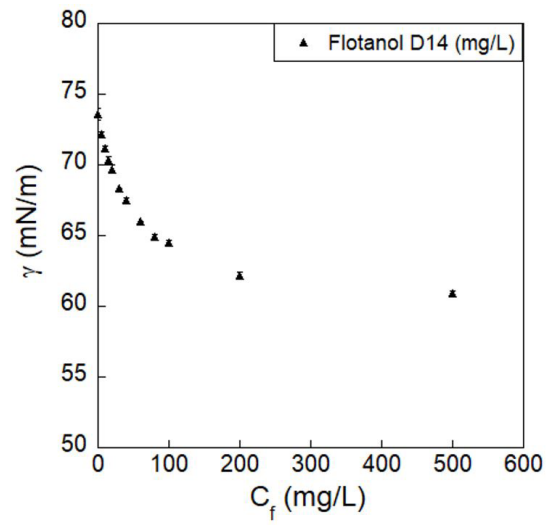

(b)

Figura 5. Curvas de holdup de gás $\left(\varepsilon_{\mathrm{g}}\right)$ em função de $\mathrm{J}_{\mathrm{g}}$ para diferentes concentrações de Flotanol DI4 (a). Tensão superficial em função da concentração do Flotanol DI4 (b).

dos dois softwares, mostrando ser uma boa opção para determinação da CCC do espumante.

A Figura 5 apresenta os resultados de medidas de holdup de gás $\left(\mathcal{E}_{\mathrm{g}}\right)$ em função da velocidade superficial do $\operatorname{ar}\left(J_{g}\right)$, para a concentração de Flotanol D I 4 variando entre 2,5 e $50 \mathrm{mg} / \mathrm{L}$. Observa-se um aumento praticamente linear do holdup com o aumento da velocidade superficial do ar, em função do aumento do número de bolhas para maiores vazões de ar, o que é intensificado com o aumento da concentração de espumante, conforme reportado por
Finch e Dobby [7]. Este resultado pode ser explicado pela redução da tensão superficial da solução de espumante na faixa estudada, conforme observado na Figura 5b, levando a uma minimização da coalescência de bolhas em função da orientação das moléculas do espumante na superfície destas bolhas (interface gás-líquido), em conformidade com diversos trabalhos da literatura $[6,7]$.

Também foi possível verificar que para um valor constante de $\int_{g}$, há um aumento de holdup com o aumento da concentração do Flotanol DI4, mesmo depois da $\mathrm{CCC}\left(\mathrm{C}_{\mathrm{f}}>7 \mathrm{mg} / \mathrm{L}\right)$, podendo 
ser explicado pelo aumento do número de bolhas, principalmente de bolhas mais finas [20], conforme visualizado na Figura 2, onde observa-se um aumento de bolhas entre 0,2 a $0,4 \mathrm{~mm}$ com o aumento da concentração do Flotanol DI4.

\section{CONCLUSÕES}

Com o aumento da concentração do espumante (Flotanol DI4) foi verificada uma diminuição mais acentuada no diâmetro médio de bolha até cerca de $7 \mathrm{mg} / \mathrm{L}$, a partir da qual a redução no diâmetro médio de bolha foi muito menos significativa, sugerindo essa como sendo a concentração crítica de coalescência (CCC). As medidas de holdup mostraram que ocorreu um aumento desse parâmetro com o aumento da velocidade superficial do ar e da concentração do Flotanol D I4.
Os dois softwares utilizados para calcular o diâmetro médio de Sauter apresentaram resultados similares, sendo que o Image/ sempre apresentou valores um pouco mais elevados de tamanho de bolhas comparado com o APBS. Ficou evidenciado a importância do controle da etapa de processamento das imagens digitais para medida do tamanho de bolha, na comparação dos softwares utilizados, mostrando que o ImageJ foi o mais confiável para este propósito.

\section{Agradecimentos}

Os autores agradecem ao CNPq pelo financiamento das bolsas de estudo do Estevão e da Renata, e ao CETEM/MCTIC pela infraestrutura.

\section{REFERÊNCIAS}

I Alexander D, Manlapig E, Bradshaw D, Harbort G. Froth flotation. In: Wills BA, Napier-Munn TJ. Mineral processing technology. 7th ed. USA: Elsevier Science \& Technology Books; 2006.

2 Rubio J, Souza ML, Smith RW. Overview of flotation as a wastewater treatment technique. Minerals Engineering. 2002; 15:139-155.

3 Sivamohan R. The problem of recovering very fine particles in mineral processing - a review. International Journal of Mineral Processing. 1990;28:247-288.

4 Aquino JA, Oliveira MLM, Fernandes MD. Flotação em coluna. In: Luz AB, Sampaio JA, Almeida SLM. Tratamento de minérios. 5. ed. Rio de Janeiro: CETEM/MCT; 2010.

5 Cho YS, Laskowski JS. Effect of flotation frothers on bubble size and foam stability. International Journal of Mineral Processing. 2002;64:69-80.

6 Couto HJB, Nunes DG, Neumann R, França SCA. Micro-bubble size distribution measurements by laser diffraction technique. Minerals Engineering. 2009;22:330-335.

7 Finch JA, Dobby GS. Column flotation. USA: Pergamon Press; 1990.

8 Grau RA, Laskowski JS, Heiskanen K. Effect of frothers on bubble size. International Journal of Mineral Processing. 2005;76:225-233.

9 Leiva J, Vinnett L, Contreras F, Yianatos J. Estimation of the actual bubble surface area flux in flotation. Minerals Engineering. 2010;23:888-894.

10 Rodrigues RT, Rubio J. New basis for measuring the size distribution of bubbles. Minerals Engineering. 2003;16:757765.

I I Maldonado M, Desbiens A, Poulina É, del Villar R, Riquelme A. Automatic control of bubble size in a laboratory flotation column. International Journal of Mineral Processing. 2015;141:27-33.

12 Gulden SJ, Riedele C, Rollié S, Kopf M-H, Nirschl H. Online bubble size analysis in micro flotation. Chemical Engineering Science. 2018;185:168-181.

13 Rodrigues RT. Desenvolvimento da técnica LTM-BSizer para a caracterização de bolhas e a avaliação de parâmetros no processo de flotação [dissertação]. Porto Alegre: Universidade Federal do Rio Grande do Sul; 2004.

14 Abramoff MD, Magelhaes PJ, Ram SJ. Image Processing with ImageJ. Biophotonics International. 2004; I :36-42.

15 Gupta AK, Banerjee PK, Mishra A, Satish P, Pradip. Effect of alcohol and polyglycol ether frothers on foam stability, bubble size and coal flotation. International Journal of Mineral Processing. 2007;82: I 26-I 37.

16 Laskowski JS. Fundamental properties of flotation frothers. In: Lorenzen L, Bradshaw DJ. Proceedings 22nd International Mineral Processing Congress; 2003 November 23-26; Cape Town, South Africa. Marshalltown: South African Institute of Mining \& Metallurgy; 2003. p. 788-797. 
17 Braga AS, Pinto TCS, Matai PHLS, Leal LS Fo. Concentração micelar crítica e concentração crítica de coalescência de reagentes de flotação. Holos. 2015;7:140-I 47.

18 Veras MM, Baltar CAN, Paulo JBA, Leite, JYP. Comparative study of the main flotation frothers using a new HYDROMESS adapted technique. Rem: Revista Escola de Minas. 2014;67(I):87-92.

19 Mazahernasab R, Ahmadi R. Determination of bubble size distribution in a laboratory mechanical flotation cell by a laser diffraction technique. Physicochemical Problems of Mineral Processing. 2016;52(2):690-702.

$20 \mathrm{Kuan} \mathrm{SH}$. The effect of solids on gas holdup, bubble size and water overflow rate in flotation [dissertation]. Montreal: McGill University; 2009.

Recebido em: 17 Jul. 2018

Aceito em: I4 Abr. 2019 